\title{
CDISC Study Reference Class
}

National Cancer Institute

\section{Source}

National Cancer Institute. CDISC Study Reference Class. NCI Thesaurus. Code C147271.

This special purpose SDTM class contains further descriptions of study-specific identifiers that will be used in subject based domains. (CDISC) 